\title{
Correlation of Serum Ferritin and Calcium Level with Febrile Seizures: A Hospital-Based Prospective Case-Control Study
}

Hamideh SHAJARI ${ }^{\mathrm{a}}$, Ahmad SHAJARI ${ }^{\mathrm{b}}$, Hamideh AZIZKHAN ${ }^{\mathrm{b}}$, Reyhaneh BARZEGARI

aDepartment of Neonatology, Shariati Hospital, School of Medicine, Tehran University of Medical Sciences, Tehran, Iran

bepartment of Pediatrics Nephrology, Ali-ebne-Abitaleb School of Medicine, Islamic Azad University, Yazd branch, Yazd, Iran

'Islamic Azad University, Yazd Branch, Yazd, Iran

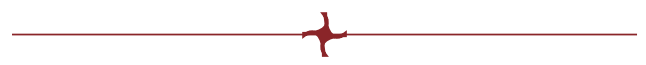

\begin{abstract}
-ABSTRACT
Background: Febrile seizure is one of the most common seizure disorders occurring in children. In the literature, there are contradicting results about the role of iron and calcium deficiency in febrile seizures that has a relatively higher prevalence. So, this study was performed to investigate the correlation of serum ferritin and calcium levels with febrile seizures in children.

Materials and methods: This case-control study was performed on a total number of 150 children aged 6 to 60 months who were frequently admitted to Shohada-e-Kargar Hospital in Yazd, Iran, due to fever from April 2017 to November 2017. The case group consisted of 49 patients with febrile seizures and the control group included 101 febrile children without seizures. Data regarding age, sex, past medical and family history of seizures, serum ferritin, and calcium levels were collected from patient medical records and laboratory results.

Results: Participants in the case and control groups had a mean age of 28.22 months and 28.16 months, respectively. Of all participants, $56 \%$ were males. Serum ferritin level was $40.61 \mathrm{ng} / \mathrm{mL}$ in the case group and $41.80 \mathrm{ng} / \mathrm{mL}$ in the control group, with no significant difference being observed between groups $(P$ value $=0.743)$. Calcium levels were $9.50 \mathrm{mmol} / \mathrm{L}$ in the case group and $9.59 \mathrm{mmol} / \mathrm{L}$ in the control group, with no significant difference being observed between groups ( $P$ value $=0.564$ ). The findings of the present research indicated that factors including age, sex, past medical and family history of seizures did not affect the mean serum levels of ferritin and calcium of the study population.

Conclusion: Based on our results, no significant difference could be established between serum ferritin and calcium levels in neither the case group nor the control group, and plausibly, these elements appear to be neither a protective nor a risk factor for a febrile seizure.
\end{abstract}

Keywords: febrile seizure, serum ferritin, calcium, fever.

\footnotetext{
Address for correspondence:

Ahmad Shajari, $M D$

Associate Professor of Pediatrics Nephrology, Ali-ebne-Abialeb School of Medicine, Islamic Azad University, Yazd Branch, Yazd, Iran

Tel.: +9803531871000

Email:a_shajari@iauyazd.ac.ir
}

Article received on the $23^{\text {rd }}$ of April 2021 and accepted for publication on the $15^{\text {th }}$ of September 2021 


\section{INTRODUCTION}

1 febrile seizure is the most common seizure disorder in about $2.5 \%$ of children with an intact nervous system (1). It most often occurs in children between the ages of six months and five years with a fever greater than $38^{\circ} \mathrm{C}$. Moreover, it is pertinent to mention that factors such as central nervous system (CNS) infections, metabolic disorders and past medical history of seizures must be ruled out to diagnose this disorder effectively $(1,2)$. The exact cause of febrile seizures is unknown, but genetic and environmental factors are speculated to play some role (3). Furthermore, factors such as genetics, nutrition, geography, associated infections, metabolic status, history of head trauma, and serum level of some minerals take part developing of seizures. Still, a clear pattern of how they are involved has not been deciphered yet. In addition, the role of serum iron levels in developing seizures has been a controversial topic in the literature so far (4). Also, febrile seizure mainly occurs at the age of 14-18 months. Besides febrile seizure, iron deficiency anemia is the most common nutritional deficiency in the first years of a child's life, indicating that febrile seizure and iron deficiency might be related (5). Iron is an essential micronutrient that is used by almost every cell in the body, and presently, iron deficiency anemia is the most common nutritional deficiency in the world. It is well established that iron is a cofactor for many enzymes in the body and plays a vital role in the production and function of neurotransmitters, along with hormonal function and DNA transcription (6).

Ferritin is a biological protein produced by animals, plants, and even bacteria. This protein plays a role in storing iron inside the cell by binding to iron (7). In humans, ferritin is a protein that binds to excess iron in the body and releases it when needed. Serum ferritin level is proportional to the body's iron stores and indicates iron stores in cells. It is the best single test for detecting iron deficiency. Ferritin decreases in iron deficiency anemia and increases in iron overload, and its levels are related to the body's total iron stores and are helpful for assessing it. Intracellular calcium regulates many essential neural activities such as neurotransmitter release, excitability, synaptic transmission, and cell viability (8). In the study carried by Kobrinsky et al, iron deficiency increased the threshold for seizures (9). In contrast, Daoud et al found that the mean hemoglobin, hematocrit, and ferritin were lower in the case group (10). In some studies, the prevalence of anemia was similar in patients with and without seizures. Elsewhere (11), serum calcium levels were lower in children with febrile seizures than controls (healthy children without fever and febrile children without seizures).

Considering the conflicting results related to the effect of iron and calcium deficiency with febrile seizures and the relatively higher prevalence of this disease, the present study was performed to determine whether there was a correlation of serum iron and calcium levels with febrile seizures in children admitted to Shohada-e-Kargar Hospital in Yazd.

\section{MATERIAL AND METHOD}

This case-control study was conducted in Shohada-e-Kargar Hospital in Yazd, Iran, from April 2017 to November 2017. A total of 150 children aged 6 to 60 months, who were frequently admitted due to fever, were selected. There were 49 children with febrile seizures in the case group and 101 febrile patients without seizures in the control group. Children in the two groups were age- and sex-matched. After the initial treatment, stabilization of vital signs and seizure control were done in the case group, and blood samples were taken from all patients. Also, information about age, sex, past medical history of seizures, family history of epilepsy was recorded in a pre-designed checklist. Additionally, serum ferritin and calcium levels were measured by ECLLA method and spectrophotometry, respectively. Parents were informed about the research process, and a written consent was obtained from them. They were assured that their information would be kept confidential and would only be used for research purposes. Noteworthily, patients did not bear any cost to participate in this research.

\section{Statistical analysis}

The present study reports the mean serum levels of ferritin and calcium and their standard deviations (SD). Also, a T-test was used to analyze the difference of serum biomarkers across the study 
groups. A significance value was considered when $\mathrm{P}<0.05$. All data obtained in the present study were analyzed in SPSS v.17 software package.

\section{Ethical considerations}

This study was performed after receiving approval from the Ethics Committee of Ali Ibn Abitaleb Medical School of Islamic Azad University, Iran. The study protocol was approved by the Islamic Azad University of Medical Sciences, Branch of Yazd, Yazd, Iran.

\section{RESULTS}

Datients included in this study had a mean age of $28.18 \pm 15.46$ months. Among all participants, 84 (56\%) patients were boys and 66 (44\%) girls. The two study groups were matched in terms of age and sex. Based on the results ob-

TA B LE 1. Patient demographic data

\begin{tabular}{lllll}
\hline & & Case & Control & P value \\
\hline Age & & $28.22 \pm 14.23$ & $28.16 \pm 16.09$ & 0.983 \\
\hline Sex & Male & $25(51 \%)$ & $59(58 \%)$ & \multirow{2}{*}{0.392} \\
\cline { 2 - 4 } & Female & $24(49 \%)$ & $42(41.6 \%)$ & \\
\hline History of seizure & $11(22.4 \%)$ & $4(4 \%)$ & 0.001 \\
\hline Family history of epilepsy & $9(18.4 \%)$ & $6(5.9 \%)$ & 0.038 \\
\hline
\end{tabular}

TA B LE 2. Patients' ferritin and calcium levels

\begin{tabular}{llll}
\hline & Case & Control & P value \\
\hline Ferritin $(\mathbf{n g} / \mathbf{m L})$ & $40.61 \pm 17.19$ & $41.81 \pm 22.27$ & 0.743 \\
\hline Calcium $(\mathbf{m m o l} / \mathbf{L})$ & $9.5 \pm 1.4$ & $9.59 \pm 0.63$ & 0.564 \\
\hline
\end{tabular}

tained from the present study, it was established that the chances of having febrile seizures would significantly increase $(p<0.05)$ in patients with a past medical or family history, particularly if their siblings had also suffered from seizures in the past. A summary of patients' demographic data can be seen in Table 1 .

The mean serum ferritin level was $40.61 \mathrm{ng} / \mathrm{mL}$ in febrile patients with seizures and $41.80 \mathrm{ng} / \mathrm{mL}$ in those without seizures. Also, the mean serum calcium level was $9.5 \mathrm{mmol} / \mathrm{L}$ in febrile patients with seizures and $59.9 \mathrm{mmol} / \mathrm{L}$ in those without seizures. However, the results showed that there was no significant difference in the mean levels of ferritin and calcium between the two groups $(p<0.05)$, as shown in Table 2.

The results obtained by us indicate that the mean level of serum ferritin in patients included in the case and control groups showed no significant difference across the groups of 6 to 23 months and 24 to 60 months. Furthermore, ferritin and calcium levels did not show a significant difference between the two groups for both sexes. Also, past medical and family history of seizures in the two study groups had no significant effect on serum ferritin and calcium levels (Table 3 ).

\section{DISCUSSION}

febrile seizure, also called febrile convulsion, is one of the most frequently occurring seizure disorders in children, and most often it occurs between the ages of six months and five years. A mandatory characteristic is that it occurs in the context of fever, with the body temperature being $38^{\circ} \mathrm{C}$ or above. Factors such as CNS infection, metabolic disorders, and history of sei-

TAB LE 3. Detailed information about patients' ferritin and calcium levels

\begin{tabular}{|c|c|c|c|c|c|c|c|}
\hline & & \multicolumn{3}{|c|}{ Ferritin $(\mathrm{ng} / \mathrm{mL})$} & \multicolumn{3}{|c|}{ Calcium $(\mathrm{mmol} / \mathrm{L})$} \\
\hline & & Case & Control & P value & Case & Control & P value \\
\hline \multirow[t]{2}{*}{ Age } & $6-23$ & $40.22 \pm 18.39$ & $35.76 \pm 19.12$ & 0.357 & $9.73 \pm 0.58$ & $9.50 \pm 0.58$ & 0.121 \\
\hline & $24-60$ & $40.92 \pm 16.50$ & $48.20 \pm 23.73$ & 0.162 & $9.31 \pm 1.81$ & $9.70 \pm 0.67$ & 0.184 \\
\hline \multirow[t]{2}{*}{ Sex } & Male & $40.40 \pm 18.66$ & $44.55 \pm 26.10$ & 0.473 & $9.66 \pm 0.61$ & $9.64 \pm 0.57$ & 0.89 \\
\hline & Female & $40.83 \pm 15.90$ & $37.92 \pm 14.82$ & 0.458 & $9.32 \pm 1.90$ & $9.52 \pm 0.71$ & 0.55 \\
\hline \multirow[t]{2}{*}{ Past history } & Yes & $40.90 \pm 19.71$ & $41 \pm 22.64$ & 0.994 & $9.77 \pm 0.79$ & $9.47 \pm 0.82$ & 0.535 \\
\hline & No & $40.52 \pm 16.69$ & $41.83 \pm 22.37$ & 0.745 & $9.42 \pm 1.53$ & $9.60 \pm 0.63$ & 0.336 \\
\hline \multirow{2}{*}{$\begin{array}{l}\text { Family history } \\
\text { of seizure }\end{array}$} & Yes & $32.44 \pm 12.82$ & $47.66 \pm 27.03$ & 0.163 & $9.48 \pm 0.66$ & $9.7 \pm 0.76$ & 0.552 \\
\hline & No & $42.45 \pm 17.64$ & $41.43 \pm 22.05$ & 0.796 & $9.5 \pm 1.52$ & $9.59 \pm 0.63$ & 0.645 \\
\hline
\end{tabular}


zures must be eliminated to diagnose this disorder effectively $(1,2)$. Additionally, other important factors such as genetic status, nutritional geography, associated infections, metabolic status, history of head trauma, and serum levels of some minerals are known to contribute to seizures, but no specific pattern of how they are involved has been discovered yet. Serum iron levels represent a highly debated item (4). Iron is a vital micronutrient used by almost every cell in the human body. It is well understood that iron is a cofactor for many enzymes in the body and plays an important role in the production of neurotransmitters and their function, as well as hormonal function and DNA transcription (6). Serum ferritin is the best single test for iron deficiency. Ferritin levels represent the total iron stores of the body and are helpful in its assessment. Intracellular calcium also regulates many essential neuronal activities, including the release of neurotransmitters, neuronal excitability, and synaptic transmission (8).

According to the results premised on the findings of the present study, patients included in the case group did not differ significantly from those in the control group in terms of ferritin and calcium serum levels. Also, age, sex, past medical and family history of seizures had no significant effect on febrile seizures. In the present study, the amount of ferritin was found to be lower in the case group than the control one. However, this relationship was not found to be significant, which is probably due to the heterogeneity of the number of samples in the two study groups.

Some scientists have reported anemia as a risk factor for febrile seizure. Some have declared anemia as a protective factor and others have found no correlation between anemia and febrile seizure. Heratipour et al's research concluded that the mean level of ferritin and other iron indices in the case group was significantly higher than the control group (12). In their study, lower iron levels were suggested as a protective factor in the prevention of febrile seizures. Abbaskhanian et al found a serum ferritin level of $89.2 \mathrm{ng} / \mathrm{mL}$ and $76.9 \mathrm{ng} / \mathrm{mL}$ in the case and control groups, respectively, and a serum iron level of 73.4 and 49.8 in the case and control groups, respectively, with a significant difference being observed (13); in their study, $40 \%$ of patients in the case group and $60 \%$ of controls had anemia; they also deduced that in cases of iron deficiency, the chances of de- veloping febrile seizures were lower, and iron deficiency could play a protective role in the occurrence of seizures. On the contrary, the pediatric patients included in our case and control groups showed no significant difference in terms of ferritin levels. Hence, no protective role of iron deficiency was detected in this study.

On the other hand, anemia was also shown to be a risk factor for febrile seizure. In this regard, Pisacane et al (14) and Rehman et al found that serum ferritin levels were significantly lower in their case group, and concluded that children with iron deficiency anemia were more likely to have seizures (30). In another study performed by Daoud et al, the mean level of hemoglobin, hematocrit, and ferritin was significantly lower in patients with febrile seizures (10). Similarly, Naseer et al reported that serum iron and hemoglobin levels were significantly lower in the case group (15). The findings of Zareifar et al's study on 500 children revealed that ferritin levels were lower in the case group, with the rate of anemia being $56.6 \%$ in the case group and $24.8 \%$ in the control group; the authors concluded that low levels of both serum ferritin and iron are risk factors for seizure fever (16). In the research of Koksal et al and Ghasemi et al, low levels of plasma ferritin and iron were also mentioned as risk factors for febrile seizures (17). Therefore, there are conflicting findings of the association between ferritin level and the occurrence of febrile seizure. Based on results obtained in this study, we found ferritin neither a protector nor a stimulator for a febrile seizure. To extend our investigation about the association of other serum chemical elements on febrile seizures, we studied calcium. The findings of Amouian et al's study showed that the levels of total iron binding capacity (TIBC) and magnesium were lower in children with febrile seizures than control subjects. On the other hand, levels of calcium, zinc, iron and, ferritin were higher in children with febrile seizures than controls (18). However, we found no significant difference between children with febrile seizure and control subjects in terms of calcium level. Further, a case-control study led by Sharavat et al about risk factors associated with the first episode of febrile seizures concluded that low serum levels of calcium, sodium, blood sugar, and the presence of microcytic hypochromic anemia were all involved in the first episode of febrile seizures (19). 
In contrast to the present study, Zargarian et al showed that serum levels of calcium, sodium, magnesium, and zinc were significantly lower in the case group (20). In the present study, the mean level of calcium was $9.5 \mathrm{mmol} / \mathrm{mL}$ in the case group and $9.6 \mathrm{mmol} / \mathrm{mL}$ in the control group. Still, in Zargarian et al's analysis, the mean level of calcium was $8.75 \mathrm{mmol} / \mathrm{mL}$ and $9.2 \mathrm{mmol} / \mathrm{mL}$ in the case and control groups, respectively.

In a study conducted by Naseer et al on 200 children (15), serum calcium levels did not significantly differ between the two study groups $(9.2 \mathrm{mmol} / \mathrm{mL}$ in the case group and $9.1 \mathrm{mmol} / \mathrm{mL}$ in the control group). Similarly to the present study, no significant difference in serum calcium levels were found in the two groups of Seyedzadeh et al's research (21). Also, the study of Rutter et al showed that levels of serum calcium and magnesium in people with febrile seizures were not different from normal people (22).

A study by Michael et al, which assessed glucose, calcium, sodium, potassium, urea, creatinine, blood cell counts, urine samples and brain scans in patients with febrile seizures, revealed that the levels of calcium and other electrolytes were in a normal range; also, the authors noted that diagnostic tests were only helpful when ordered based on patient's condition or medical history (23). The results of Ellyn et al suggested that sodium and calcium levels did not significantly differ in febrile patients with or without seizures and had no relationship with the rates of seizure recurrence. According to this study and the American Academy of Pediatrics practice parameter, routine electrolyte evaluations are not recommended for the first episode of febrile seizure (24). In another study by Mohammadi et al, no association was observed between electrolyte disturbances in the form of an increase or decrease in sodium, potassium, calcium, or blood sugar and the onset of a febrile seizure, and the decision about measuring the particular serum electrolyte was made by assessing the individual patient's condition (25).

The conflicting results of these studies can be caused by several factors. First, the sample size and number of samples in case and control groups are different. Also, children's nutritional status and their use of iron supplements in other communities can cause conflicting results in studies. The present study had some limitations. One of them was that the history of iron supplementation and nutritional status was not evaluated in the studied patients. Another one was that blood indices, including hemoglobin and MCV, were also not evaluated. However, unlike other studies, the effect of past medical and family history of seizures, age and sex on serum ferritin and calcium levels were evaluated in the present research.

Several studies regarding the impact of different biological factors on the occurrence of febrile seizure have been published. Nevertheless, results are heterogenous, which is partly explained by methodological differences. In the future, larger studies should be conducted in order to get reliable conclusions.

\section{CONCLUSION}

Considering the results of the present study and comparing them to those reported by other authors, it is not recommended to perform exhaustive tests for patients who are admitted for fever but do not have seizures. In addition, clinicians should consider that exhaustive tests are not needed in case of simple febrile seizures in well-appearing children returning to a normal baseline after a febrile seizure. It is important to exclude different possible alternative diagnosis if other signs are present (meningeal signs or other clinical features that could suggest a CNS infection). We found that, in children with febrile seizures, calcium and ferritin evaluation and electroencephalography and other diagnostic methods do not seem necessary unless a patient has symptoms of electrolyte disorder or a past medical history of seizures.

However, more extensive controlled studies with larger sample sizes are recommended to elucidate the effect of electrolytes and iron deficiency anemia in children as well as their impact on febrile seizures.

Conflicts of interest: none declared.

Financial support: none declared.

Acknowledgments: We thank all patients for their participation in our study. 


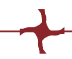

\section{References}

1. Marcdante K, Kliegman RM. Nelson essentials of pediatrics e-book. Elsevier Health Sciences, 2014.

2. Arzimanoglou A, Aicardi J. The epilepsy of Sturge-Weber syndrome: clinical features and treatment in 23 patients. Acta Neurol Scand Suppl 1992;140:18-22.

3. Derakhshanfar $\mathrm{H}$, Abaskhanian $\mathrm{A}$, Alimohammadi H, ModanlooKordi M. Association between iron deficiency anemia and febrile seizure in children. Med Glas (Zenica) 2012;9:239-242.

4. Billoo AG. Association between iron deficiency anemia and febrile seizures. J Coll Physicians Surg JCPSP 2005;15:338-340.

5. Hartfield DS, Tan J, Yager JY, et al. The association between iron deficiency and febrile seizures in childhood. Clin Pediatr (Phila) 2009;48:420-426.

6. Hartfield D. Iron deficiency is a public health problem in Canadian infants and children. Paediatr Child Health 2010;15:347-350.

7. Arosio P, Elia L, Poli M. Ferritin, cellular iron storage and regulation. IUBMB Life 2017;69:414-422.

8. Hui L, Geiger NH, Bloor-Young $D$, et al. Release of calcium from endolysosomes increases calcium influx through $\mathrm{N}$-type calcium channels: Evidence for acidic store-operated calcium entry in neurons. Cell Calcium 2015;58:617-627.

9. Kobrinsky NL, Yager JY, Cheang MS, et al. Does iron deficiency raise the seizure threshold?

J Child Neurol 1995;10:105-109.

10. Daoud AS, Batieha A, Abu-Ekteish F, et al. Iron status: a possible risk factor for the first febrile seizure.

Epilepsia 2002;43:740-743.

11. Namakin $K$, Zardast $M$, Sharifzadeh G, et al. Serum Trace Elements in Febrile Seizure: A Case-Control Study. Iran J Child Neurol [Internet] 2016;10:57-60. Available from: https://pubmed.ncbi.nlm.nih. gov/27375757

12. Haratipor $H$, Sohrabi MB, Zolfaghari $P$, et al. The comparison of serum iron, ferritin and TIBC in children aged 9 months to 6 years with febrile seizure. Knowledge and Health 2014;9:55-60.

13. Abbaskhanian A, VahidShahi K, Parvinnezhad N. The association between iron deficiency and the first episode of febrile seizure. JBUMS 2009;11:32-36.

14. Pisacane A, Sansone R, Impagliazzo N, et al. Iron deficiency anaemia and febrile convulsions: case-control study in children under 2 years. BMJ 1996;313:343-344.

15. Naseer MR, Patra KC. Correlation of serum iron and serum calcium levels in children with febrile seizures. Int J Contemp Pediatr 2017;2:406-410.

16. Zareifar $S$, Hosseinzadeh HR, Cohan N. Association between iron status and febrile seizures in children. Seizure 2012;21:603-605.

17. Köksal AO, Özdemir O, Büyükkaragöz $B$, et al. The association between plasma ferritin level and simple febrile seizures in children. J Pediatr Hematol Oncol 2016;38:512-516.
18. Amouian $S$, Mohammadian $S$, Behnampour N, Tizrou M. Trace elements in febrile seizure compared to febrile children admitted to an academic hospital in Iran, 2011. J Clin Diagnostic Res 2013;7:2231.

19. Sharawat IK, Singh J, Dawman L, Singh A. Evaluation of risk factors associated with first episode febrile seizure. J Clin Diagnostic Res 2016;10:SC10.

20. Bidar S, Zargarian T. Relationship between serum levels of sodium, potassium, calcium, magnesium, zinc febrile seizure.

Journal of Birjand University of Medical Sciences 2018;40(10).

21. Sayedzadeh SA, Hemati M. Serums Sodium \& Calcium Level in Children with Simple and Recurrent Febrile Convulsion. J Kermanshah Univ Med Sci 2007;10:e81821.

22. Rutter N, Smales OR. Calcium, magnesium, and glucose levels in blood and CSF of children with febrile convulsions.

Arch Dis Child 1976;51:141-143.

23. Gerber MA, Berliner BC.

The Child With a'Simple'Febrile Seizure: Appropriate Diagnostic Evaluation. Am J Dis Child 1981;135:431-433.

24. Thoman JE, Duffner PK, Shucard JL. Do serum sodium levels predict febrile seizure recurrence within 24 hours? Pediatr Neurol 2004;31:342-344.

25. Mohammadi J. Biochemical disorders in children with febrile seizure. J ILAM Univ Med Sci 2009;16:25-30. 